\title{
Modeling Thermal Effects in Metals Irradiated by Copper Nanoclusters
}

\author{
Balt Batgerel $^{3}$, Stefka Dimova², Temenujka Kupenova ${ }^{4}$, Igor Puzynin ${ }^{1}$, Taisia Puzynina ${ }^{1}$, Ivan \\ Hristov $^{2}$, Radoslava Hristova ${ }^{2}$, Zafar Tukhliev ${ }^{1}$, and Zarif Sharipov ${ }^{1, \star}$ \\ ${ }^{1}$ Joint Institute for Nuclear Research, 141980, Dubna, Russia \\ ${ }^{2}$ Sofia University "St. Kliment Ohridski", Sofia, Bulgaria \\ ${ }^{3}$ Mongolian State University of Science and Technology, Ulaanbaatar, Mongolia \\ ${ }^{4}$ Institute for Nuclear Research and Nuclear Energy, Bulgarian Academy of Sciences, Sofia, Bulgaria
}

\begin{abstract}
One of the promising areas in condensed matter physics is the study of the interaction of nanoclusters with various metallic targets. In this paper we investigate the thermal processes in metals irradiated with nanoclusters by the molecular dynamics method. In the framework of this method wave effects of the heat transfer are obtained, a feature which is absent in the thermal spike model. We repost results of computer simulation of the structural changes in irradiated copper targets of different sizes depending on the energy (in the range of 10-50 eV/atom) of copper nanoclusters.
\end{abstract}

\section{Introduction}

Over the past few decades, a growing number of theoretical and experimental works has been done to study the interaction of heavy ions, pulsed ion beams and nanoclusters with condensed media. These studies are motivated by the possibility of applications to the creation of radiation-resistant materials, thin coatings, new materials and technologies. Among these studies one of the most important and promising is the simulation of the interaction of nanoclusters with metallic targets [1]. When nanoclusters interact with a metal target, a large amount of energy is released in a small volume when calculated per unit volume, which leads to structural changes. The changes can be a consequence of thermal processes or of elastic interactions (repulsion of target atoms). The experimental studies give the final result of structural changes in the targets. The application of methods of mathematical modeling makes it possible to see the development of the whole process of the target irradiation in time. Note that in the real life experiments the target irradiation with nanoclusters consists of two distinct stages: the formation and the acceleration of the nanoclusters. This is technically a very difficult task. Therefore, the mathematical modeling remains an important tool and feasible alternative for such studies. In particular, the problem of modeling the accelerated nanoclusters considered in [2]. The purpose of this work is to study by the molecular dynamics (MD) method $[1,3]$ the thermal processes in metals irradiated with nanoclusters. The modeling of the irradiation with nanoclusters of various targets is quite similar to modeling the irradiation by other sources, e.g., heavy ions of high energies and/or pulsed beams. When heavy ions of high energies are used, a large amount of energy

\footnotetext{
^e-mail: zarif@jinr.ru
} 
per unit volume is released in the local target region. Due to the inelastic interactions at high energies of the incident heavy ion, the direct application of the MD method for the problem of high-energy ion irradiation results in unreliable predictions. Nevertheless, the irradiation of targets with pulsed ion beams can approximately be simulated as irradiation with nanoclusters sequences and in this case the problem can be also tackled within the framework of the MD method.

\section{Formulation of the problem}

The methods of classical molecular dynamics are based on the model representation of a multiparticle system in which all particles (atoms or molecules) are material points. The behavior of an individual particle is described by the classical Newton's equations of motion $[1,3]$, which can be written as:

$$
m_{i} \frac{d^{2} \mathbf{r}_{i}}{d t^{2}}=\mathbf{f}_{i}
$$

Here $i$ is the particle number $(1 \leq i \leq N) ; N$ is the total number of particles; $m_{i}$ is the particle mass; $\mathbf{f}_{i}$ is the resultant of all forces acting on the particle, having the following representation:

$$
\mathbf{f}_{i}=-\frac{d U\left(\mathbf{r}_{1}, \ldots, \mathbf{r}_{N}\right)}{d \mathbf{r}_{i}}+\mathbf{f}_{i}^{\text {ex }},
$$

where $U$ is the interaction potential between the particles; $\mathbf{f}_{i}^{\text {ex }}$ is the force due to external fields (not present in the reported simulations).

In this work we study the thermal processes in a copper target irradiated with copper nanoclusters $\mathrm{Cu}_{(141)}$ of energies $10-50 \mathrm{eV} /$ atom.

\section{Method of numerical solution and results}

Currently, there is a number of existing and widely used software packages for the application of the methods of molecular dynamics. In our investigations and computer experiments we have used LAMMPS [4].

The simulation of the process of interaction of copper nanoclusters with the target was carried out in the following way. Within the framework of the molecular-dynamic approach, we solve the problem of irradiating a copper target with nanoclusters of energy 10-50 eV/atom. Further, using the obtained solution, we calculate the temperature profiles of the target as a function of time. To do this, the target material is divided into cells and inside each cell the temperature at each instant time $t_{k}$ is computed. It is to be observed that the number of particles in the cell must be larger than 100 . Thus, we get the temperature distribution in the form $T_{\mathrm{md}}\left(x_{i}, y_{i}, z_{i}, t_{k}\right)$. Within the framework of molecular dynamics, the computational domain was a parallelepiped with sides $22 \times 22 \times 16 \mathrm{~nm}$, the number of particles in the target was 439200 and in the nanoclusters it was 141. As the interatomic potential, the universal EAM (embedded atom, [4]) potential for copper, integrated into LAMMPS package, was used. The calculation area for the temperature is divided into $55 \times 55 \times 30$ cells (in the $x$-, $y$-, $z$-directions respectively).

Figure 1 shows the crater dynamics formation as a function of time in a target irradiated with copper nanoclusters of energy $50 \mathrm{eV} /$ atom. It can be seen from the figure that within the time frame from 5 ps to 20 ps the shape of the crater does not change significantly.

Figure 2 presents the dynamics of the changes (in time) of the target temperature field at a depth of $5 \mathrm{~nm}$. The figure illustrates the development of a thermal process. Namely, at time $0.7 \mathrm{ps}$ at a depth of $5 \mathrm{~nm}$ in a circle with a diameter of $7 \mathrm{~nm}$, the temperature increases (the highest temperature rise is 

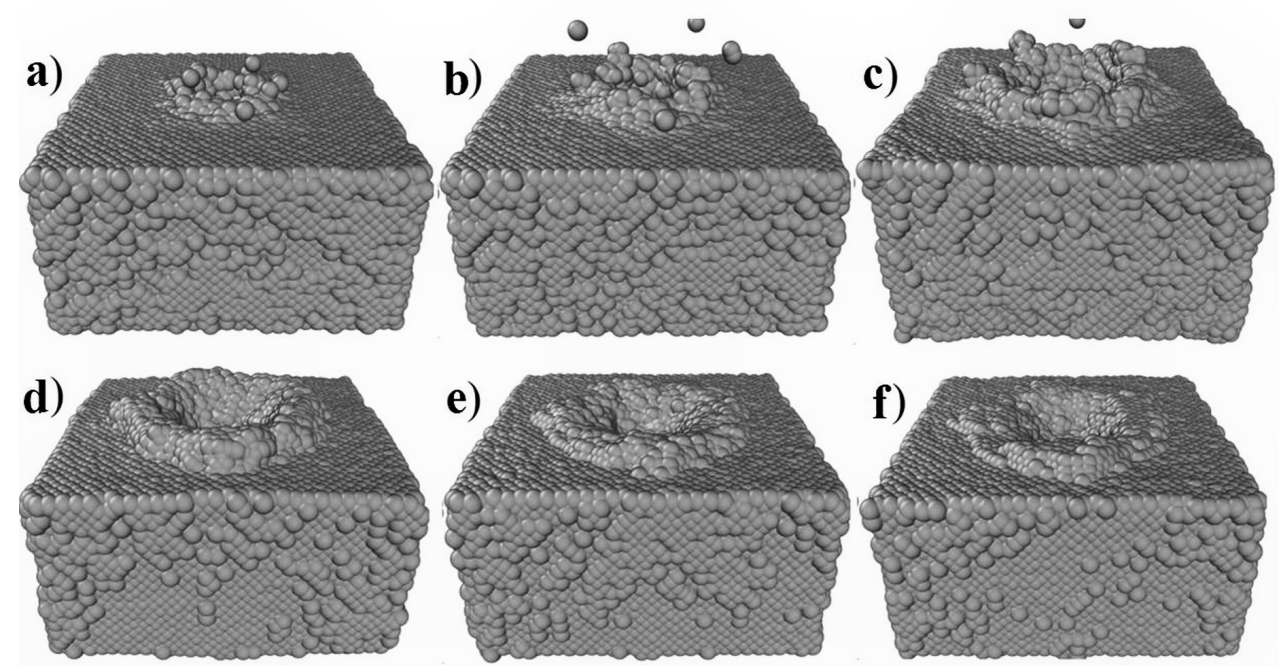

Figure 1. Dynamics of crater formation as a function of time: a) $0.5 \mathrm{ps}$; b) 1 ps; c) 2 ps; d) 5 ps; e) 10 ps; f) 20 ps

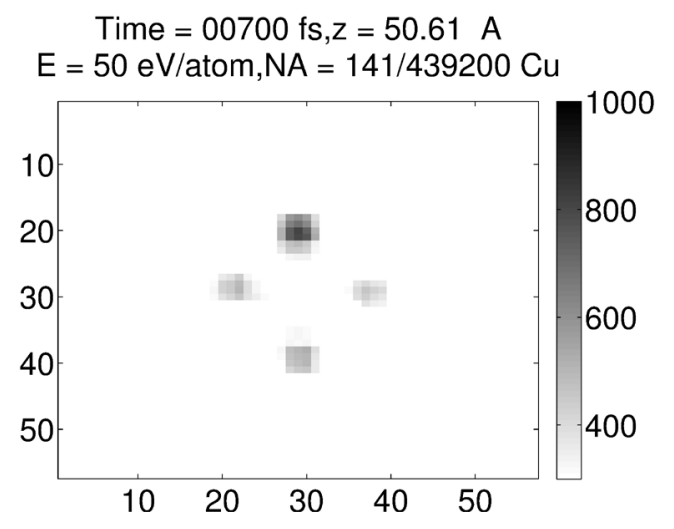

a)

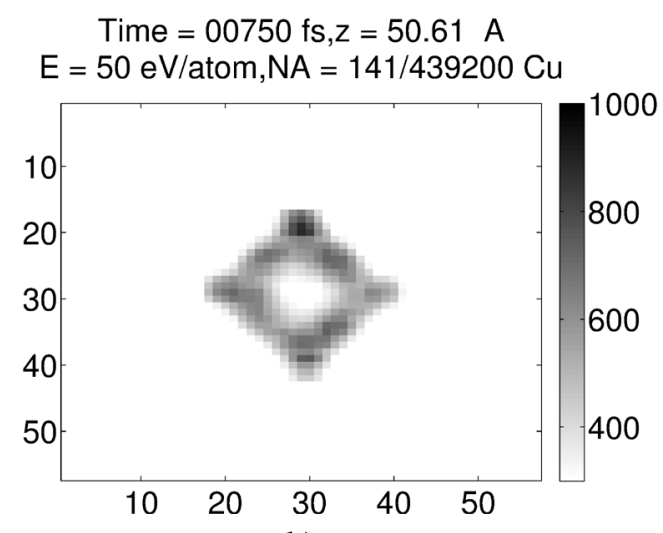

b)

Time $=01000 \mathrm{fs}, \mathrm{z}=50.61 \mathrm{~A}$
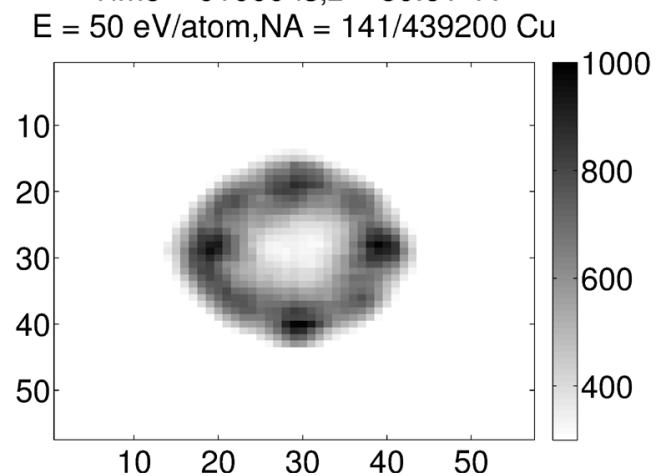

c)

Time $=01120 \mathrm{fs}, \mathrm{Z}=50.61 \mathrm{~A}$
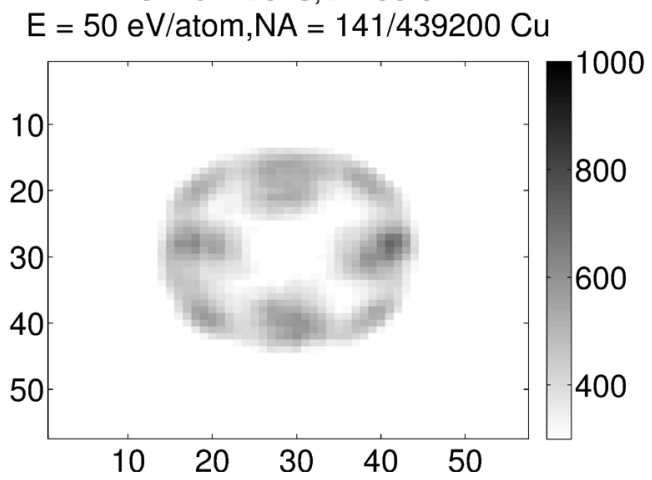

d)

Figure 2. Dynamics of changes in the temperature field of the target at a depth of $5 \mathrm{~nm}$ as a function of time a) $700 \mathrm{fs}$; b) $750 \mathrm{fs}$; c) $1000 \mathrm{fs}$; d) $1120 \mathrm{fs}$ 
along the OX and OY axes). Similar results are observed at different depths and times. They point to the occurrence of a wave-like thermal conductivity directed along the principal crystallographic axes of the lattice. Fractal analysis methods, [5], were used for processing the results.

\section{Conclusion}

We simulated the interaction of copper nanoclusters of energy $10-50 \mathrm{eV} /$ atom with a copper target in the framework of the molecular dynamics method and using the software package LAMMPS. Based on the computer simulations, we can draw two main conclusions:

1. The molecular dynamics approach predicts the occurrence of heat transfer wave effects, phenomena which are absent when heat equations of parabolic types are solved.

2. The resulting heat transfer wave effects are of anisotropic: the wave transport of the heat depends on the principal crystallographic axes of the crystal lattice.

\section{Acknowledgements}

The work was financially supported by the RFBR grant No. 17-01-00661-a and partially supported by a grant of the Plenipotentiary Representative of the Republic of Bulgaria at the JINR. The work of I. Hristov, R. Hristova, S. Dimova is partially supported by the NSF of BMSE under Grant DFNI-I02/9 and Grant DFNI-I02/8.

\section{References}

[1] B.Batgerel, A.Yu.Didyk, I.V.Puzynin et al., Journal of Surface Investigation 9, 1026-1030 (2015)

[2] B.Batgerel, E.G.Nikonov, I.V.Puzynin, Vestnik RUDN, Journal of Mathematics, Information Sciences and Physics 1, 47-51 (2014)

[3] H.T. Holmurodov, I.V.Puzynin et al., PEPAN 34, 2, 472 (2003)

[4] S. Plimpton, Journal of Computational Physics 117, 1-19 (1995)

[5] B.B. Mandelbrot, The Fractals Geometry of Nature (N. Y., Freeman, 1982) 468 pp. 